Research Article

\title{
Comparative study of anaphylaxis incidence in patients receiving anti- snake venom with or without prophylactic adrenaline: a prospective, randomized, blinded study
}

\author{
Raghuraman M. S. ${ }^{1 *}$, Sivaraman B. ${ }^{2}$
}

\begin{abstract}
${ }^{1}$ Department of Emergency Medicine, Mahatma Gandhi Medical College and Research Institute, Puducherry, India ${ }^{2}$ Department of Emergency Medicine, Rajah Muthiah Medical College, Chidambaram, Tamilnadu, India
\end{abstract}

Received: 13 May 2016 Accepted: 11 June 2016

*Correspondence to:

Dr. Raghuraman M. S., Email: drraghuram70@ gmail.com

Copyright: () the author(s), publisher and licensee Medip Academy. This is an openaccess article distributed under the terms of the Creative Commons Attribution NonCommercial License, which permits unrestricted noncommercial use, distribution, and reproduction in any medium, provided the original work is properly cited.

\begin{abstract}
Background: Anti-snake venom serum (ASV) administered for snake-bite patients is the most specific treatment available. Unfortunately, it can cause severe anaphylaxis which can be fatal sometimes.

Methods: Fifty patients were included in the study and randomly divided into group A, who received sub-cutaneous adrenaline before administration of antivenom serum and group B who did not. Each group comprised of twenty-five patients. The incidences as well as the severity of anaphylaxis in both groups and also the effects of subcutaneous adrenaline on the hemodynamic were analysed.

Results: Anaphylactic reactions were significantly less in group A than group B population $(\mathrm{p}<0.05)$. Hemodynamic variables such as blood pressure, pulse rate have responded similarly in both the groups ( $>>0.05)$ implying that administration of sub-cutaneous adrenaline is safe.

Conclusions: Prophylactic subcutaneous adrenaline before anti snake venom administration is highly efficacious and safe in reducing the incidence as well as severity of anaphylactic reactions.
\end{abstract}

Keywords: Snake bites, Antivenins, Anaphylaxis, Adrenaline

\section{INTRODUCTION}

The most specific treatment available for snake bite envenomation is administration of polyvalent anti- snake venom serum (ASV). While it is effective in neutralizing the circulating venom, it can cause allergic reactions. The reactions can be of two types, early or late. The magnitude of early reactions may vary from mild itching, nausea, vomiting to severe systemic anaphylaxis like bronchospasm, shock, angioedema occurring from 10 minutes to 3 hours following anti-venom administration. ${ }^{1}$ The early reactions are not IgE mediated, but are most likely caused by complement activation by $\mathrm{IgG}$ aggregates in the horse serum. ${ }^{2,3}$ Although we do not know the exact incidence of early allergic reactions following administration of ASV, it varies between 5 to $80 \%{ }^{4}$ It is 2 to $50 \%$ according to Malasit $\mathrm{P}$ et al. ${ }^{2}$ This variation in the incidence is understandable because of many factors like the geo-graphical area, type of antivenom, various manufacturing methods etc. What is more worrying is, it can be fatal sometimes. ${ }^{5}$ The early reactions could not be predicted by skin or conjunctival sensitivity tests. ${ }^{2}$ Indeed, these tests may pre-sensitize the patient and cause greater risk. ${ }^{6}$ Technological 
modification of anti-venom with pepsin or papain can attenuate but not abolish acute reactions. ${ }^{7}$

The main aim of this study was to assess the role of prophylactic sub-cutaneous adrenaline in reducing the anaphylactic reactions in patients receiving polyvalent anti snake venom and its safety on the hemodynamics. The anti-venom commonly used in our country is the polyvalent type covering the common four venomous snakes namely the Cobra, Krait, Russel's viper and sawscaled viper. It is prepared from equine serum and lyophilized. This study was taken up because of the high incidence of early adverse reactions following ASV administration. It was approximately $50 \%$ in South India at that time although the exact incidence was not known. According to personal experience of one author in our geo-graphical area, it was $60 \%{ }^{4}$ The incidence of severe anaphylaxis was about $20 \%$ in the department of emergency medicine of the institute where this study was conducted.

\section{METHODS}

After approval from the ethical committee of the institute, this study was conducted on 50 adult patients randomly divided into two groups of 25 patients each as per the inclusion/exclusion criteria to assess the role of prophylactic sub-cutaneous adrenaline in reducing the incidence of anaphylactic reactions in patients receiving polyvalent anti snake venom as well as its safety. This study was conducted in the Emergency room at a medical college in south India, from September 2011 to August 2013.

Group A -patients receiving polyvalent anti-snake venom with prophylactic sub-cutaneous adrenaline. $(0.25 \mathrm{ml}$ of 1:1000 dilution).

Group B - patients receiving polyvalent anti snake venom without prophylactic sub-cutaneous adrenaline.

Informed written consent was obtained from the close relative of the patient. Patients were allotted to either one of the groups by block randomization method. Out of fifty four eligible patients, fifty patients were included in the study and four patients were excluded as per the criteria.

The second author, who had done the study, was blinded to the administration of adrenaline. So, this was an investigator blinded (single blinded) study. In view of ethical issues, the control group did not receive any placebo (single arm study).

The efficacy and safety of prophylactic sub-cutaneous adrenaline in polyvalent anti-snake venom receiving patients of group A has been analysed by comparing with the group B patients.

\section{Inclusion criteria}

- All poisonous snake-bite patients receiving polyvalent anti snake venom

- Adult patients of either sex aged 13-60 years.

\section{Exclusion criteria}

- Known hypertensive patients

- Known cardiac and other valvular heart disease patients

- Recent cerebro-vascular accident patients of less than 6 months duration

- Known atopic patients.

\section{Investigations}

\section{Coagulation profile}

- $\quad$ Bleeding time

- Clotting time

- Activated partial thromboplastin time

- Whole blood clotting time

Electrocardiogram (ECG)

Renal functions test and serum electrolytes

\section{Monitors}

- Continuous cardiac monitoring

- Non-invasive blood pressure monitoring

- Respiratory rate monitoring

- Pulse oximetry

- Temperature monitoring.

The pulse rate, systolic and diastolic blood pressure, respiratory rate, oxygen saturation, temperature were noted before administration of ASV as baseline parameters and every ten minutes during the first hour and every twenty minutes during the next two hours following administration of ASV in both group of patients, because the peak effect of subcutaneous adrenaline would be well within three hours. The mean values of systolic blood pressure, diastolic blood pressure and pulse rate of patients of both groups were analysed statistically to assess the safety of adrenaline on the hemodynamics.

In addition to the above parameters, all the patients were looked for any early adverse reactions following three hours of ASV administration, because the early adverse reactions are very common during the three hours following anti-venom administration. ${ }^{1}$ The observed adverse reactions were graded as mild, moderate and severe as per the following classification. ${ }^{5}$ The rescue measures in case of adverse reactions were done as per the emergency room protocols like temporary stopping of ASV infusion, administration of intravenous antihistamines and steroids, intramuscular or intravenous adrenaline, airway management etc. 


\section{Classification of acute adverse reactions}

The acute adverse reactions of anti-venom serum, classified according to the following criteria. ${ }^{5}$

- $\quad$ Mild

- Moderate

- Severe

Mild reactions include pruritis, skin rash, mild urticaria, fever, rigors, nausea, vomiting, cough, and light headedness.

Moderate reactions include extensive urticaria, facial edema, bronchospasm without cyanosis and hoarseness of voice.

Severe reactions include pulmonary edema, bronchospasm with cyanosis, stridor and shock.

All the parameters have been subjected to appropriate statistical analysis in Epi-info software. Percentages, mean, standard deviation were used as descriptive statistics and Chi-square, Mann-Whitney $U$ test were used for inferential statistics.

\section{RESULTS}

Out of 25 participants in each group, $60 \%$ were male and $40 \%$ were female in group A, whereas in group B, $48 \%$ were male and $52 \%$ were female. The highest percentage of snake bite was observed in 21 to 30 years of age in both group A (36\%) and group B (32\%). Mostly unknown species were responsible for snake bite in both the groups. The percentages were 68 and 60 respectively for group A and B. The most common site of snake bite was extremities, comprising $88 \%$ in group $\mathrm{A}$ and $100 \%$ in group B. Fang mark was observed in $64 \%$ of sample in group A and 60\% of sample in group B. The arrival of the study population to emergency room following snake bite between 1 to 6 hours constitutes maximal percentage in both the groups (i.e.) $60 \%$ and $64 \%$ respectively for group $\mathrm{A}$ and group $\mathrm{B}$.

There was significant difference in anaphylactic reactions between groups demonstrated by Pearson Chi-square test $(p=0.048)$. Anaphylactic reactions were significantly less among those received prophylactic sub-cutaneous adrenaline $(12 \%)$ as compared to those who did not receive $(48 \%)$. In group A population, the occurrence of anaphylactic reactions were very less. Only $8 \%$ of patients developed mild anaphylactic reactions and $4 \%$ of patients developed moderate form of anaphylactic reactions in group A. Interestingly, none of the group A population developed severe form of anaphylactic reactions. Whereas, in group B, $32 \%$ of patients developed mild anaphylactic reactions, $12 \%$ developed moderate anaphylactic reactions and $4 \%$ developed severe form of anaphylactic reactions (Figure 1).

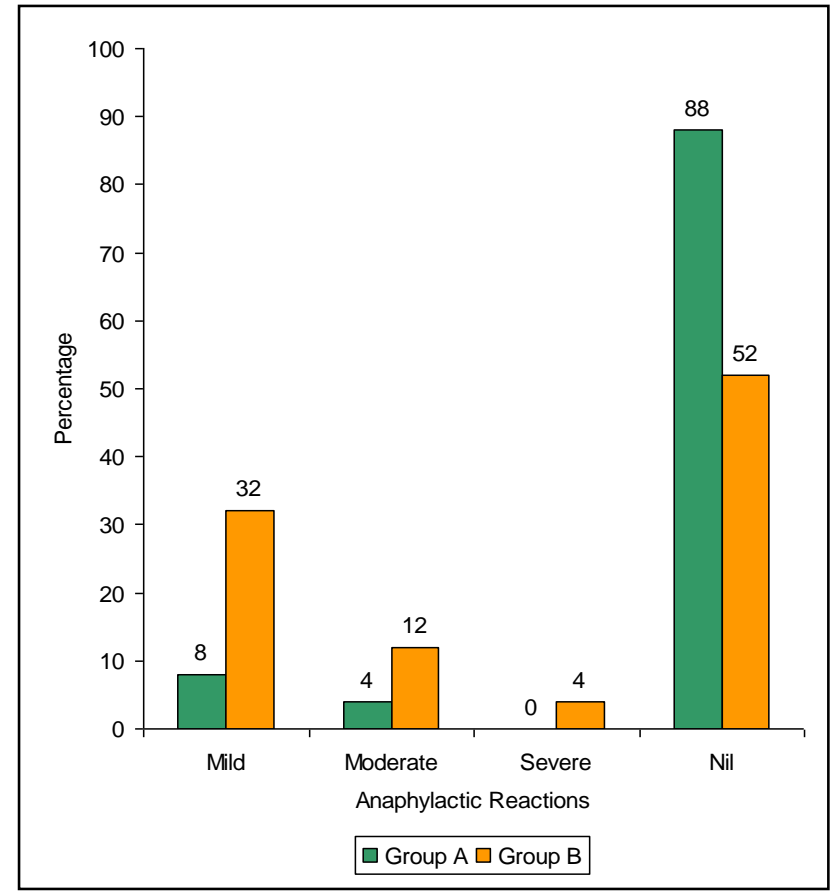

$\mathrm{P}=0.048$

Figure 1: Comparison of incidences of anaphylactic reactions between groups.

Hemodynamic variables such as blood pressure, pulse rate have responded similarly in both the groups during three hours following ASV administration, as demonstrated by Mann-Whitney ' $U$ ' test ( $>0.05)$, implying that administration of sub-cutaneous adrenaline did not cause significant change in pulse rate, blood pressure thereby ensuring its safety as well (Table $1,2,3$ ).

Table 1: Hemodynamic variables at 1 hour MannWhitney ' $U$ ' test.

\begin{tabular}{|ll|l|}
\hline Variables & Z Value & P Value \\
\hline Systolic blood pressure & 0.848 & 0.397 \\
\hline Diastolic blood pressure & 0.556 & 0.579 \\
\hline Pulse rate & 0.447 & 0.655 \\
\hline
\end{tabular}

Table 2: Hemodynamic variables at 2 hours-MannWhitney ' $U$ ' Test.

\begin{tabular}{|lll|}
\hline Variables & Z Value & P Value \\
\hline Systolic blood pressure & 0.146 & 0.884 \\
\hline Diastolic blood pressure & 1.013 & 0.311 \\
\hline Pulse rate & 0.360 & 0.719 \\
\hline
\end{tabular}

Table 3: Hemodynamic variables at 3 hours-MannWhitney ' $U$ ' test.

\begin{tabular}{|lll|}
\hline Variables & Z Value & P Value \\
\hline Systolic blood pressure & 0.136 & 0.892 \\
\hline Diastolic blood pressure & 1.481 & 0.139 \\
\hline Pulse rate & 0.165 & 0.869 \\
\hline
\end{tabular}


None of the patients of either group required mechanical ventilation or died during the stay in the emergency room.

\section{DISCUSSION}

This was a prospective, randomized, single blinded (investigator only), single arm study, done in the emergency room, to assess the role of sub-cutaneous adrenaline in reducing the anaphylactic reactions in patients receiving polyvalent anti-snake venom. This study was conducted on 50 patients during the period of September 2011 to August 2013. Detailed evaluation was done using a semi-structured proforma.

Premawardhena AP et al done a study to assess the efficacy and safety of low dose adrenaline injected subcutaneously to prevent acute adverse reactions to polyvalent anti snake venom. They found that subcutaneous administration of $0.25 \mathrm{ml}$ of $1: 1000$ adrenaline, immediately before administration of antivenom serum to patients with envenomation after snake bite reducing the incidence of acute adverse reactions to serum. ${ }^{5}$ Williams DJ et al and De silva et al also observed the same finding in their study. ${ }^{8,9}$ They had also observed that premedication with promethazine or hydrocortisone was not effective. ${ }^{8,9}$ Amin MR et al had also observed that intra-muscular adrenaline rather than intravenous antihistamine or hydrocortisone was more effective and quicker in controlling the adverse reactions following anti-snake venom administration. Although they did not use adrenaline as premedication, they suggested that it may be employed after finding that it was very effective and rapid in onset when they had used it as rescue measure. ${ }^{10}$ In a comparative study of promethazine with placebo, Fan HW et al observed that promethazine did not reduce the incidence of adverse reactions of anti-venom serum. ${ }^{11}$ The same was also observed by Williams DJ et al and De Silva et al subsequently. ${ }^{8,9}$ This was the reason that we did not choose anti-histamine or corticosteroid for comparison.

In our study also, it has been found that there was significant reduction in the incidence of acute adverse reactions to polyvalent anti snake venom when premedicated with $0.25 \mathrm{ml}$ of $1: 1000$ adrenaline subcutaneously. In our study it has been found additionally that none of the patients in the study group A had developed severe form of anaphylactic reactions following polyvalent anti snake venom administration.

Dassanayake AS et al had done a study to assess the safety of low dose subcutaneous adrenaline given as prophylaxis against acute adverse reactions to Anti Snake Venom in patients bitten by snake. They found that low dose $(0.25 \mathrm{ml}, 1: 1000)$ subcutaneous adrenaline did not cause significant change in pulse rate or blood pressure. ${ }^{12}$

In our study also it has been found that low dose subcutaneous adrenaline did not cause significant change in blood pressure, pulse rate.
Thus, we believe that the use of $0.25 \mathrm{ml} 1: 1000$ subcutaneous adrenaline administration before the polyvalent anti snake venom administration not only reduces the occurrence of polyvalent anti snake venom induced anaphylactic reactions significantly, but also safe.

The main limitation of this study is that it was restricted to the emergency room only and the duration of stay in intensive care or general ward, other complications, final outcome etc. were not analysed.

\section{CONCLUSION}

In our study, regarding the role of prophylactic subcutaneous adrenaline in patients receiving polyvalent anti snake venom, we found the following effects of prophylactic sub-cutaneous adrenaline.

- Significant decrease in the incidence of anaphylactic reactions following polyvalent anti snake venom administration.

- None of the patients had developed the severe form of anaphylactic reactions with the usage of prophylactic subcutaneous adrenaline.

- Low dose (0.25 ml 1:1000 dilution) prophylactic subcutaneous adrenaline administration did not cause significant change in the vital parameters like blood pressure, pulse rate, respiratory rate, oxygen saturation and temperature.

We conclude that the use of adrenaline $0.25 \mathrm{ml} \mathrm{1:1000}$ dilution subcutaneously, before polyvalent anti snake venom administration is highly efficacious and safe in reducing the incidence of early adverse reactions and thereby reducing the morbidity and mortality of the patients.

\section{ACKNOWLEDGEMENTS}

The authors would like to sincerely thank Mr. G. Ezhumalai, Msc. PhD, Statistician of Sri Balaji Vidyapeeth University, India, for helping us in statistics.

Funding: No funding sources Conflict of interest: None declared

Ethical approval: The study was approved by the Institutional Ethics Committee

\section{REFERENCES}

1. Nuchprayoon I, Garner P. Interventions for preventing reactions to snake anti-venom. Cochrane Database of systematic Reviews. 1999;4:CD 002153.

2. Malasit P, Warrel DA, Chanthavanich P, Viravan C, Mongkolsapaya J, Singhthong B, et al. Prediction, prevention, and mechanism of early (anaphylactic) antivenom reaction in victims of snake bites. BMJ. 1986;292:17-20.

3. Sutherland SK. Serum reactions-an analysis of commercial anti-venoms and the possible role of anti- 
complementery activity in de-novo reactions to antivenoms and antitoxins. Medical Journal of Australia. 1977;1:613-5.

4. Mahadevan S. Drug alert, RPC (South) JIPMER, Pondicherry, INDIA. 2005;1(2):1-8.

5. Premawardhena AP, de Silva CE, Fonseka MM, Gunatilake SB, de Silva HJ. Low dose subcutaneous adrenaline to prevent acute adverse reactions to antivenom serum in people bitten by snakes: randomised, placebo controlled trial. BMJ. 1999;318:1041-1043.

6. Indian National Snakebite Protocols 2009. Available at

http://164.100.130.11:8091/nationalsnakebitemanage mentprotocol.pdf.

7. UPCC. Anti-venom therapy for snake bite. UPCC. $2001 ;(3) 1: 1-4$. Available at https://poisoncontrol.utah.edu/newsletters/pdfs/toxico logy-today-archive/Vol3_No1.pdf.

8. Williams DJ, Jensen SD, Nimorakiotakis B, Muller $\mathrm{R}$, Winkel KD. Antivenom use, premedication and early adverse reactions in the management of snakebites in rural Papua New Guinea. Toxicon. 2007;49:780-92.

9. De silva HA, Pathmeshwaran A, Ranasinha CD, Jayamanne S, Samarakoon SB, Hittharage A, et al. Low dose adrenaline, promethazine and hydrocortisone in the prevention of acute adverse reactions to anti-venom following snake bite: a randomized, double blinded, placebo controlled trial. PLOS Med. 2011;8(5):e1000435.

10. Amin MR, Mamun SMH, Rashid R, Rahman M, Ghose A, Sharmin S, et al. Anti-snake venom: use and adverse reaction in a snake bite study clinic in Bangladesh. J Venom Anim Toxins incl Trop Dis. 2008;14(4):660-72.

11. Fan HW, Marcopito LF, Cardoso JL. Sequential randomised and double blind trial of promethazine prophylaxis against early anaphylactic reactions to antivenom for bothrops snakebites. BMJ. 1999;318:1451-2.

12. Dassanayake AS, Karunanayake P, Kasturiratne KT. Safety of subcutaneous adrenaline as prophylaxis against acute adverse reactions to anti-venom serum in snakebite. Ceylon Med J. 2000;47:48-9.

Cite this article as: Raghuraman MS, Sivaraman B. Comparative study of anaphylaxis incidence in patients receiving anti-snake venom with or without prophylactic adrenaline: a prospective, randomized, blinded study. Int J Basic Clin Pharmacol 2016;5:1436-40. 